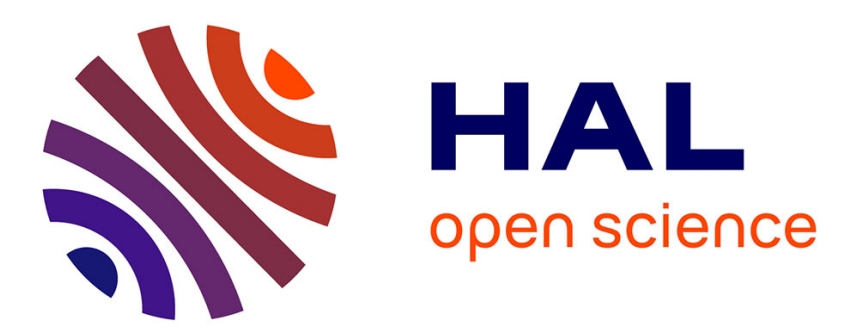

\title{
Chemical composition of the volatile oil of Laggera aurita Schulz from Burkina-Faso
}

Jean-Philippe Mevy, Anne Bousquet-Mélou, Stephane Greff, J. Millogo, Catherine Fernandez

\section{> To cite this version:}

Jean-Philippe Mevy, Anne Bousquet-Mélou, Stephane Greff, J. Millogo, Catherine Fernandez. Chemical composition of the volatile oil of Laggera aurita Schulz from Burkina-Faso. Biochemical Systematics and Ecology, 2006, 34 (11), pp.815 - 818. 10.1016/j.bse.2006.06.005 . hal-01764239

\section{HAL Id: hal-01764239 \\ https://hal-amu.archives-ouvertes.fr/hal-01764239}

Submitted on 11 Apr 2018

HAL is a multi-disciplinary open access archive for the deposit and dissemination of scientific research documents, whether they are published or not. The documents may come from teaching and research institutions in France or abroad, or from public or private research centers.
L'archive ouverte pluridisciplinaire HAL, est destinée au dépôt et à la diffusion de documents scientifiques de niveau recherche, publiés ou non, émanant des établissements d'enseignement et de recherche français ou étrangers, des laboratoires publics ou privés. 


\title{
Chemical composition of the volatile oil of Laggera aurita Schulz from Burkina-Faso
}

\author{
J.P. Mevy ${ }^{\text {a,* }}$, A. Bousquet-Mélou ${ }^{\text {a }}$, S. Greff ${ }^{\text {a }}$, J. Millogo $^{\text {b }}$, C. Fernandez ${ }^{\text {a }}$ \\ ${ }^{a}$ Laboratoire Biosystématique et Ecologie Méditerranéenne, IMEP CNRS UMR6116, \\ Université de Provence, Case 421, FST Saint-Jérôme, 13397 Marseille Cedex 20, France \\ ${ }^{\mathrm{b}}$ Laboratoire de Biologie et d'Ecologie Végétales, Université de Ouagadougou, 03 BP 7021 Ouagadougou 03, Burkina Faso
}

Keywords: Asteraceae; Laggera aurita; Essential oil; Sesquiterpenes; Monoterpenes; Phenol ether

\section{Subject and source}

The genus Laggera Sch. Bip. Ex Benth. \& Hook, belongs to the family Asteraceae and contains about 20 species widespread in tropical Africa and Asia (Zheng et al., 2003). Plants of this genus are often used in folk medicine specially for the treatment of jaundice (Mahmoud et al., 1996). Few studies have been carried out regarding the chemistry of Laggera aurita. The report published by Zutshi et al. (1975) on the composition of the volatile oil from plant samples harvested in India, revealed the presence of an aromatic ether $(2,3-$ dimethoxy-p-cymene). Because the composition of the essential oil from the genus Laggera may vary considerably according to the origin of the plant studied (Sohounhloue et al., 2004; Kuiate et al., 2002; Nebie et al., 2002), we present in this report the chemical composition of the volatile oil of L. aurita harvested in BurkinaFaso. This work also, emphasises on the chemotaxonomy of species of the genus Laggera based on their essential oil composition shown in literature reports.

Plant material was randomly collected from several specimens in September 2000, near the city of Ouagadougou (Burkina-Faso). A voucher specimen is kept at the herbarium of the University of Provence (MAR-2005-01).

\section{Previous works}

Only two phytochemical studies are available for L. aurita. The first study was conducted with specimens from Asia and was a qualitative approach and describes the occurrence of seven constituents in the volatile oil samples (Zutshi et al., 1975). The second report showed that the volatile oil was highly bactericidal (Geda, 1995).

\footnotetext{
* Corresponding author. Tel.: +3349128 88 74; fax: +3349128 8707.

E-mail address: jean-philippe.mevy@up.univ-mrs.fr (J.P. Mevy).
} 
Table 1

Chemical composition of the essential oil from leaves of Laggera aurita

\begin{tabular}{|c|c|c|}
\hline Compound & $\mathrm{RI}^{\mathrm{a}}$ & $\% \mathrm{RA}^{\mathrm{b}}$ \\
\hline Linalool & 1098 & 0.7 \\
\hline cis-p-Menth-2-en-1-ol & 1117 & 0.1 \\
\hline trans-p-Menth-2-en-1-ol & 1135 & 0.1 \\
\hline 4-Terpineol & 1173 & 0.6 \\
\hline p-Cymen-8-ol & 1182 & $\operatorname{Tr}^{\mathrm{c}}$ \\
\hline$\alpha$-Terpineol & 1187 & 0.4 \\
\hline Decanal & 1206 & 0.1 \\
\hline trans-Carveol & 1217 & $\operatorname{Tr}$. \\
\hline Nerol & 1229 & 0.1 \\
\hline Thymol methyl ether & 1235 & 0.7 \\
\hline Isothymol methyl ether & 1244 & 0.1 \\
\hline Thymol & 1299 & $\operatorname{Tr}$. \\
\hline 2,4-Decadienal & 1320 & Tr. \\
\hline$\alpha$-Methyl-3-phenylallyl alcohol & 1329 & Tr. \\
\hline Longipinene deriv. & 1350 & 5.0 \\
\hline 3-Allylguaiacol & 1362 & 0.2 \\
\hline Longicyclene & 1368 & 0.2 \\
\hline Farnesene deriv. & 1373 & 1.9 \\
\hline $6 s-2,3,8,8$-tetramethyltricyclo[5.2.2.01,6]undec-2-ene & 1379 & 1.8 \\
\hline $10 s, 11 s$-Himachala-3(12), 4-diene & 1399 & 0.3 \\
\hline Longifolene & 1404 & 0.3 \\
\hline Isocaryophyllene & 1405 & 0.2 \\
\hline$\beta$-Caryophyllene & 1422 & 9.6 \\
\hline Dimethoxy- $p$-Cymene & 1436 & 27.7 \\
\hline$\alpha$-Himachalene & 1449 & 3.0 \\
\hline$\alpha$-Humulene & 1456 & 6.4 \\
\hline Geranyl acetone & 1460 & 0.1 \\
\hline Alloaromadendrene & 1462 & 0.2 \\
\hline Bicyclo[7.2.0]undec-4-ene, 4,11,11-trimethyl-8-methylene-deriv. & 1468 & 0.3 \\
\hline 1H-Benzocycloheptene, 2,4a,5,6,9a-hexahydro-3,5,5,9-tetramethyl-, deriv. & 1478 & 2.5 \\
\hline Alloaromadendrene & 1462 & 0.2 \\
\hline Bicyclo[7.2.0]undec-4-ene, 4,11,11-trimethyl-8-methylene-deriv. & 1468 & 0.3 \\
\hline 1H-Benzocycloheptene, 2,4a,5,6,9a-hexahydro-3,5,5,9-tetramethyl-, deriv. & 1478 & 2.5 \\
\hline$\alpha$-Amorphene & 1482 & 0.2 \\
\hline 1H-Benzocycloheptene, 2,4a,5,6,9a-hexahydro-3,5,5,9-tetramethyl-, deriv. & 1484 & 0.6 \\
\hline$\beta$-Ionone & 1498 & 0.4 \\
\hline Valencene & 1496 & 0.1 \\
\hline$\beta$-Himachalene & 1500 & 0.3 \\
\hline$\alpha$-Muurolene or its enantiomere: $1 \xi, 6 \xi, 7 \xi$-Cadina-4,9-diene & 1502 & 0.3 \\
\hline$\gamma$-Cadinene & 1514 & 0.7 \\
\hline$\delta$-Cadinene & 1525 & 1.2 \\
\hline Elemol & 1537 & 0.6 \\
\hline Caryophyllene oxide deriv. & 1549 & 0.3 \\
\hline Spathulenol & 1574 & 0.2 \\
\hline Caryophyllene oxide & 1578 & 6.1 \\
\hline Humulene epoxide & 1602 & 2.9 \\
\hline Caryophylladienol I & 1628 & Tr. \\
\hline T-cadinol & 1633 & 2.4 \\
\hline Cedrene hydrate deriv. & 1636 & 0.5 \\
\hline$\alpha$-Cadinol & 1645 & 1.7 \\
\hline 2,6,9,11-Dodecatetraenal, 2,6,10-trimethyl- & 1686 & 0.5 \\
\hline ( \pm )-Phytone & 1827 & 1.5 \\
\hline Total identified & & 86.1 \\
\hline
\end{tabular}

\footnotetext{
${ }^{a}$ Retention index (RI).

b Relative area (RA).

c Traces.
} 
Table 2

The major constituents of the essential oil of several Laggera species from different origins

\begin{tabular}{|c|c|c|c|c|c|}
\hline Species & Location & Main components & RA $(\%)$ & Main groups & Reference \\
\hline \multirow[t]{2}{*}{ Laggera aurita } & India & $\begin{array}{l}n \text {-Heptacosane } \\
n \text {-Dotriacontane } \\
\delta \text {-Cadinol } \\
\text { Laggerol } \\
m \text {-Menth-6-en-8-ol } \\
\text { 2,3-Dimethoxy-p-cymene }\end{array}$ & & $\begin{array}{l}\text { Alkane } \\
\text { ox sesquiterpene } \\
\text { ox monoterpene } \\
\text { Phenolic ether }\end{array}$ & Zutshi et al. (1975) \\
\hline & Burkina-Faso & $\begin{array}{l}2,5 \text {-Dimethoxy-p-cymene } \\
\beta \text {-Caryophyllene } \\
\alpha \text {-Humulene }\end{array}$ & $\begin{array}{l}27.7 \\
9.6 \\
6.4\end{array}$ & $\begin{array}{l}\text { Phenolic ether } \\
\text { Sesquiterpene hc }\end{array}$ & Present study \\
\hline \multirow[t]{3}{*}{ Laggera alata } & Nigeria & $\begin{array}{l}2,5 \text {-Dimethoxy-p-cymene } \\
\alpha \text {-Humulene } \\
\beta \text {-Caryophyllene }\end{array}$ & $\begin{array}{l}29.17 \\
14.2 \\
10.14\end{array}$ & $\begin{array}{l}\text { Phenolic ether } \\
\text { Sesquiterpene hc }\end{array}$ & Ekundayo et al. (1989) \\
\hline & & $\begin{array}{l}\text { 2,5-Dimethoxy-p-cymene } \\
\alpha \text {-Eudesmol }\end{array}$ & $\begin{array}{l}11.17 \\
12.55\end{array}$ & $\begin{array}{l}\text { Phenolic ether } \\
\text { ox sesquiterpene }\end{array}$ & Ekundayo et al. (1989) \\
\hline & & $\begin{array}{l}\text { 2,5-Dimethoxy- } p \text {-cymene } \\
\text { Sabinene } \\
\text { 6-Hydroxycarvotanacetone-7- } O \text {-angelate }\end{array}$ & $\begin{array}{l}44 \\
16 \\
2\end{array}$ & $\begin{array}{l}\text { Phenolic ether } \\
\text { Monoterpene hc }\end{array}$ & Onayade et al. (1990) \\
\hline $\begin{array}{l}\text { Laggera alata } \\
\text { var. alata }\end{array}$ & Comoros & $\begin{array}{l}\beta \text {-Caryophyllene } \\
\alpha \text {-Muurolene } \\
\alpha \text {-Caryophyllene }\end{array}$ & $\begin{array}{l}30.5 \\
21.1 \\
16.2\end{array}$ & Sesquiterpene hc & Said Hassani et al. (2005) \\
\hline $\begin{array}{l}\text { Laggera alata } \\
\quad \text { var. montana }\end{array}$ & Cameroun & $\begin{array}{l}\text { 2,5-Dimethoxy-p-cymene } \\
\text { 7-epi- } \gamma \text {-Eudesmol } \\
\text { Juniper camphor }\end{array}$ & $\begin{array}{l}34 \\
21 \\
7\end{array}$ & $\begin{array}{l}\text { Phenolic ether } \\
\text { ox sesquiterpene }\end{array}$ & Kuiate et al. (2002) \\
\hline Laggera gracilis & Cameroun & $\begin{array}{l}2,5 \text {-Dimethoxy-p-cymene } \\
\gamma \text {-Eudesmol } \\
\beta \text {-Caryophyllene }\end{array}$ & $\begin{array}{l}33 \\
10 \\
7\end{array}$ & $\begin{array}{l}\text { Phenolic ether } \\
\text { ox sesquiterpene } \\
\text { Sesquiterpene hc }\end{array}$ & Kuiate et al. (2002) \\
\hline Laggera oloptera & Cameroun & $\begin{array}{l}\beta \text {-Caryophyllene } \\
\text { Germacrene D } \\
\text { Sabinene }\end{array}$ & $\begin{array}{l}15-20 \\
10-17 \\
2-28\end{array}$ & $\begin{array}{l}\text { Sesquiterpene hc } \\
\text { Monoterpene hc }\end{array}$ & Kuiate et al. (2002) \\
\hline & Burkina-Faso & $\begin{array}{l}\alpha \text {-Pinene } \\
\text { Limonene }\end{array}$ & $\begin{array}{l}72.1 \\
14.8\end{array}$ & Monoterpene hc & Nebie et al. (2002) \\
\hline Laggera pterodonta & Benin & $\begin{array}{l}\text { 2,5-Dimethoxy-p-cymene } \\
\text { 10-epi- } \gamma \text {-Eudesmol } \\
\text { Juniper camphor }\end{array}$ & $\begin{array}{l}30.5 \\
24.6 \\
7.5\end{array}$ & $\begin{array}{l}\text { Phenolic ether } \\
\text { ox sesquiterpene }\end{array}$ & Sohounhloue et al. (2004) \\
\hline & Cameroun & $\begin{array}{l}\text { 2,5-Dimethoxy-p-cymene } \\
\gamma \text {-Eudesmol } \\
\alpha \text {-Eudesmol } \\
\text { Juniper camphor }\end{array}$ & $\begin{array}{l}4-50 \\
17-45 \\
4-15 \\
4-12\end{array}$ & $\begin{array}{l}\text { Phenolic ether } \\
\text { ox sesquiterpene }\end{array}$ & Ngassoum et al. (2000) \\
\hline Laggera tomentosa & Endemic of Ethiopia & Chrysanthenone & 58 & ox monoterpene & Asfaw et al. (2003) \\
\hline
\end{tabular}

$\mathrm{ox}=$ oxygenated $\mathrm{hc}=$ hydrocarbon.

\section{Present study}

From dry leaves ( $200 \mathrm{~g}$ ), crude essential oil was obtained by hydrodistillation and then analysed by GC-MS (Hewlett-Packard computerized system constituted by a 6890 gas chromatograph coupled to a Hewlett-Packard 5973N quadrupole mass spectrometer) using a HP5MS capillary column $(30 \mathrm{~m} \times 0.25 \mathrm{~mm} \times 0.25 \mu \mathrm{m}$ id $)$. The oven temperature was programmed at $50{ }^{\circ} \mathrm{C}$ (initial temperature maintained $2 \mathrm{~min}$ ) to reach $160{ }^{\circ} \mathrm{C}$ at a rate of 
$2{ }^{\circ} \mathrm{C} / \mathrm{min}$, kept constant $5 \mathrm{~min}$, then programmed to reach $270{ }^{\circ} \mathrm{C}$ at a rate of $50{ }^{\circ} \mathrm{C} / \mathrm{min}$ where it remained for $6 \mathrm{~min}$. Carrier gas (Helium 99.95\%) was set at a constant flow of $1 \mathrm{ml} / \mathrm{min}$ during run. A $2-\mu 1$ sample volume was injected with automatic injector ALS7683 in a split mode (purge flow set to $50 \mathrm{ml} / \mathrm{min}$ after $1 \mathrm{~min}$ ). Identification was achieved by components mass spectrum comparison to those of personal library, and confirmed by retention indices expected by literature (Adams, 1989; Kuiate et al., 2002).

Hydrodistillation of dry leaves of L. aurita, afforded orange oil with a yield of $0.05 \%$ (w/w) on dry weight basis. A 1000 -fold dilution in hexane of essential oil analysis led to the identification of 52 constituents representing about $86 \%$ of the composition of the oil. The relative amounts of the volatile constituents identified are shown in Table 1. The oil is characterized by a high percentage of sesquiterpene hydrocarbons $(33 \%)-\beta$-caryophyllene $(9.6 \%)$ $\alpha$-humulene $(6.4 \%)$ being the major components - and phenolic ethers $(28.5 \%)$ - the dominant one being dimethoxy-p-cymene (27.7\%). In the L. aurita specimen analysed from India, only seven of the eight constituents separated were identified, namely; $n$-heptacosane, $n$-dotriacontane, laggerol, $\delta$-cadinene, 2,3-dimethoxy- $p$-cymene, $\alpha$-cadinol and $m$-menth-6-en-8-ol (Zutshi et al., 1975). The sesquiterpene alcohol (laggerol) was not found in our oil samples. $\mathrm{C}_{27}$ and $\mathrm{C}_{32}$ alkanes could not be detected because of their high molecular weight and GC analysis parameters employed. These variations in the distribution of constituents suggest the existence of two chemical races of L. aurita.

\section{Chemotaxonomic significance}

Several other Laggera species have been studied regarding their volatile oil composition (Table 2). Based on the major components characterized from these plants, two chemotaxonomic groups may be established:

Group 1: Species that mainly synthesize the phenol ether dimethoxy-p-cymene and sesquiterpenic constituents. This was described from such species as Laggera pterodonta (Ngassoum et al., 2000), L. pterodonta (Sohounhloue et al., 2004), Laggera gracilis (Kuiate et al., 2002), Laggera alata (Ekundayo et al., 1989) and L. alata var. montana (Kuiate et al., 2002) harvested, respectively, from Cameroun, Benin, Cameroun, Nigeria and Cameroun. In this group, we may also include species that major constituents are phenol ethers and monoterpene hydrocarbons as L. alata from Nigeria (Ekundayo et al., 1989).

Group 2: Species producing principally either monoterpenes or sesquiterpenes or both classes of terpenic components, except phenyl ether. This is the case for Laggera tomentosa, an endemic plant of Ethiopia in which main volatile oil constituents are oxygenated monoterpenes (Asfaw et al., 2003). While Laggera oloptera from Burkina-Faso affords mainly monoterpene hydrocarbons (Nebie et al., 2002), both monoterpene and sesquiterpene constituents were shown as the dominant components identified from Cameroun specimens (Kuiate et al., 2002).

On this basis, our L. aurita sample from Burkina-Faso may be included in the group $\mathrm{n}^{\circ} 1$ together with L. gracilis, L. alata, L. pterodonta and L. alata var. montana.

\section{References}

Adams, R.P., 1989. Identification of Essential Oil by Ion Trap Mass Spectroscopy. Academic Press, Inc., San Diego, USA. Asfaw, N., Storesund, H.J., Aasen, A.J., 2003. J. Essent. Oil Res. 15, 102.

Ekundayo, O., Oguntimein, B., Laakso, I., Hiltunen, R., 1989. Planta Med. 55 (6), 573.

Geda, A.K., 1995. Fat Sci. Technol. 97 (12), 458.

Kuiate, J.-R., Bessiere, J.-M., Zollo, P.-H.A., 2002. Flavour Fragrance J. 17, 105.

Mahmoud, A.M., Mohamed, O.K., Mohamed, A.K., Abdel, M.B.El.A., Hassan, A.R.M., El Tahir, I.M., 1996. Sudan: Country Report to the FAO International Technical Conference on Plant Genetic Resources, Leipzig.

Nebie, R.H.Ch., Belanger, A., Millogo-Rasolodimby, J., Yameogo, R., Sib, F.S., 2002. J. Soc. Ouest-Afr. Chim. $14,143$.

Ngassoum, M.B., Jirovetz, L., Buchbauer, G., Fleischhacker, W., 2000. J. Essent. Oil Res. 12, 345.

Onayade, O.A., Scheffer, J.J.C., Schripsema, J., 1990. Flavour Fragrance J. 5, 165.

Said Hassani, M., Said Abdallah, B., Zrira, S., Benjilali, B., 2005. J. Essent. Oil-Bear. Plants 8 (1), 15.

Sohounhloue, K.D., Sagbo, A.U., Menut, C., Bessiere, J.-M., 2004. J. Essent. Oil Res. 16 (3), 193.

Zheng, Q., Xu, Z., Sun, X., Yao, W., Sun, H., Cheng, C.H.K., Zhao, Y., 2003. Phytochemistry 63, 835.

Zutshi, S.K., Bamboria, B.K., Bokadia, M.M., 1975. Curr. Sci. 44, 571. 